\section{Fatores associados à não-adesão ao tratamento com anti-hipertensivos em pessoas atendidas em unidades de saúde da família}

\author{
Risk factors associated with non-adherence to \\ anti-hypertensive medication among patients \\ treated in family health care facilities
}

${ }^{1}$ Centro de Ciências da Saúde, Fundação Universidade Regional de Blumenau, Blumenau, Brasil. ${ }_{2}^{2}$ Faculdade de Medicina Universidade de São Paulo, São Paulo, Brasil.

Correspondência E. T. Santa-Helena Departamento de Medicina Centro de Ciências da Saúde Fundação Universidade Regional de Blumenau. Rua Antonio da Veiga 140, Blumenau, SC 89012-900, Brasil. erntsh@furb.br

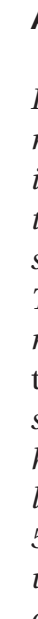

\begin{abstract}
In order to estimate the prevalence of treatment non-adherence and associated factors among individuals with systemic arterial hypertension treated at family health care facilities, a crosssectional study was performed with 595 patients. The dependent variable non-adherence was measured with a Medication Adherence Questionnaire (MAQ). A hierarchical logistic regression model was used to analyze socioeconomic, health care-related, personal, and treatment-related variables. Prevalence of non-adherence was 53\%. Variables associated with non-adherence were: (1) socioeconomic - belonging to economic classes $C, D$, or E; work market participation in unskilled labor; (2) health care-out-of-pocket payment for medication; more than six months since last physician consultation; and (3) personal and treatment characteristics - previous interruption of treatment; being on treatment for less than three years; and presence of a common mental disorder. The study of determinants of non-adherence articulated in a hierarchical model suggests that social inequalities are either directly associated with non-adherence or mediated by personal and health services factors.
\end{abstract}

Hypertension; Medication Adherence; Primary Health Care; Epidemiologic Studies
Ernani Tiaraju de Santa-Helena 1 Maria Ines Battistella Nemes 2 José Eluf Neto 2

\section{Introdução}

As doenças do aparelho circulatório foram responsáveis por mais de 800 mil internações processadas pelo Sistema Único de Saúde (SUS) em 2006, com um custo aproximado de 1 bilhão e 300 milhões de Reais e se mantêm como principal causa agrupada de mortes no Brasil. A Região Sul apresenta a maior taxa de internações por doenças cardiovasculares (86 por 10 mil habitantes em 2006), os custos médios de internação mais elevados, e as maiores taxas brutas de mortalidade do país (Departamento de Informática do SUS. http://www.datasus.gov.br, acessado em 15/Mai/2007).

A hipertensão arterial sistêmica (HAS) é importante causa direta ou participante da morbidade e mortalidade por doenças do aparelho circulatório. Revisão sistemática de 44 estudos em 35 países estimou que acometa pouco mais $30 \%$ da população adulta, com predomínio no sexo masculino ${ }^{1}$. No Brasil, estudo de revisão de prevalência de HAS em diversas cidades encontrou variações de $19 \%$ a $44 \%$ (que dependem do critério adotado e processo de aferição), com freqüências mais elevadas entre os maiores de 60 anos e os de menor escolaridade 2 . O Estado de Santa Catarina conta com cerca de 200 mil pessoas com HAS cadastradas no programa Hiperdia do Ministério da Saúde ${ }^{3}$.

O controle da HAS depende de medidas dietéticas e de estilo de vida (atividade física regular, 
combate ao tabagismo, controle do consumo de álcool) e, quando necessário, do uso regular de medicamentos. No entanto, estima-se que um terço das pessoas regularmente acompanhadas em serviços de saúde tem sua pressão arterial mantida em níveis desejáveis. A insuficiente adesão ao tratamento medicamentoso é apontada como um dos importantes determinantes desse problema 4,5 .

Os serviços de saúde da família da rede de atenção primária à saúde no Brasil são responsáveis pela assistência a milhões de pessoas com HAS. Poucos estudos analisaram a adesão dos pacientes ao tratamento no contexto assistencial. Prado et al. 6, em amostra de 109 pessoas atendidas em unidade de atenção primária em Florianópolis, Santa Catarina, obtiveram 68\% de não-adesão utilizando contagem manual de comprimidos. Ungari 7, utilizando um questionário, estimou não-adesão de $54 \%$ entre hipertensos de alguns núcleos de saúde da família em Ribeirão Preto, São Paulo.

A não-adesão é classicamente considerada um fenômeno complexo e multideterminado. Os estudos epidemiológicos apontam variados fatores associados à não-adesão que costumam ser analisados conjuntamente, valorizando-se as associações que permanecem no modelo multivariado final. O uso de modelos saturados, entretanto, pode eventualmente ocultar o poder explicativo de uma ou um conjunto de variáveis. Isso ocorre particularmente com aquelas variáveis indicativas de condições que, não obstante seu poder explicativo, são menos imediatamente relacionadas a um comportamento específico de tomada de medicamentos, como, por exemplo, a escolaridade. Modelos hierárquicos, utilizados em outros objetos epidemiológicos se mostraram uma alternativa de abordagem que permitiu uma melhor compreensão dos fenômenos estudados 8 .

O objetivo deste estudo é analisar a associação de múltiplos fatores sócio-econômicos, médico-assistenciais, pessoais e do tratamento medicamentoso, hierarquicamente ordenados, com a não-adesão em pessoas com HAS, assistidas em unidades de saúde da família no Município de Blumenau, Santa Catarina.

\section{Métodos}

A população de estudo foi composta pelas pessoas com HAS moradoras nas áreas de abrangência das unidades de saúde da família de Blumenau.

Para estimar a associação de possíveis fatores de risco à não-adesão foram utilizados como parâmetros: poder estatístico de $80 \%$, erro alfa de 5\%, razão de não expostos/expostos 3:1, freqüência de não-adesão de $45 \%$ entre os não-expostos e razão de prevalência de 1,3, resultando em 608 pessoas. Foram acrescentados $10 \%$ para prever possíveis perdas ou recusas, totalizando 667 pessoas.

A amostragem foi realizada em dois estágios. No primeiro estágio, foram sorteadas 10 unidades de saúde da família (dentre as 34 existentes que estavam em atividade há mais de 6 meses). No segundo estágio, foram identificadas as pessoas moradoras da área de abrangência cadastradas como hipertensas nas unidades sorteadas. Procedeu-se então à amostragem aleatória estratificada, distribuindo-se o tamanho amostral proporcionalmente entre as pessoas com HAS cadastradas em cada unidade.

A variável dependente foi a não-adesão ao tratamento com medicamentos anti-hipertensivos. A prevalência de não-adesão foi medida pelo Questionário de Adesão a Medicamentos-Equipe Qualiaids (QAM-Q) que consta de três perguntas e resulta em uma medida composta, em que somente é considerado aderente o indivíduo que relata ter tomado $80 \%$ a $120 \%$ das doses prescritas, de modo correto (sem "feriados", "tomada errática”, "meia-adesão” ou abandono), e relata que sua pressão arterial estava normal na última aferição 9 .

As variáveis de estudo, escolhidas e ordenadas com base no modelo teórico apresentado na Figura 1 foram: (1) sócio-econômicas: raça/ cor (referida pelo entrevistado), escolaridade (categorizada em anos de estudo 0, 1-4, 5-8, 9-12 ou mais), renda individual, ocupação, situação ocupacional, classe econômica, condições de moradia (bloco 1); (2) assistência dos serviços de saúde: tempo desde a última consulta, comparecimento a consultas, consulta em prontosocorro ou internação hospitalar nos últimos 12 meses, tipo de serviço que presta assistência, fonte de obtenção dos medicamentos (bloco 2); (3) características pessoais: sexo, idade (em anos completos), estado civil, religião, consumo atual de tabaco, consumo atual de álcool, prática de atividades físicas, interrupção prévia do tratamento, satisfação com o serviço de saúde, outras doenças clínicas associadas, transtornos mentais comuns (bloco 3); (4) tratamento medicamentoso: número de medicamentos, número de anti-hipertensivos, esquema posológico e classes de medicamentos utilizados, relato de reações adversas, tempo de tratamento (em meses), (bloco 3).

A ocupação foi codificada e agrupada com base na Classificação Brasileira de Ocupações (CBO, http://www.mtecbo.gov.br, acessado em 06/Jun/2006). 
Figura 1

Modelo explicativo para a não-adesão.

Bloco 1

STATUS SÓCIO-ECONÔMICO

(raça/cor, escolaridade, ocupação, situação ocupacional, renda, condições de moradia, classe econômica)

Bloco 2

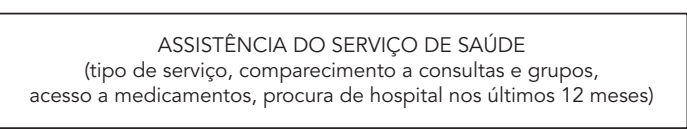

Bloco 3

CARACTERISTICAS PESSOAIS

Estilo de vida: estado civil, atividade física,

consumo de álcool, tabaco; Crenças e comportamentos:

Satisfação com serviço, interrupções prévias, religião:

Demográficas e da doença: sexo, idade,

tempo de doença e tratamento,

outras doenças, complicações

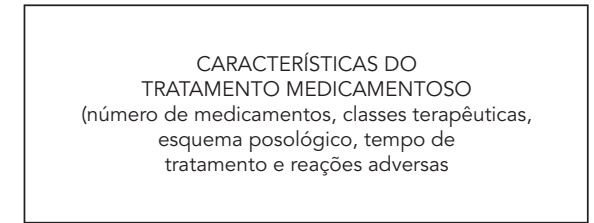

NÃO-ADESÃO
A classe econômica foi construída com base nos Critérios de Classificação Econômica do Brasil (Associação Brasileira de Empresas de Pesquisa. http://www.abep.org/codigoguias/ abep_cceb.pdf, acessado em 20/Nov/2004), que tem por referência a escolaridade e os bens de consumo.

Utilizou-se o Self-Reporting Questionnaire (SRQ-20) para rastreamento de transtornos mentais comuns na atenção primária 10 .

A satisfação com o serviço de saúde foi estimada por 8 questões gerais previamente validadas 11 que se referiam à estrutura e ao acesso à unidade (2 questões), atendimento, tratamento e cuidado da equipe (3 questões), satisfação com tratamento e com médico (2 questões), satisfação geral (1 questão). Constituiu-se um índice de satisfação geral, que foi obtido pela composição dos escores médios das 8 questões, sendo um a "máxima satisfação" e quatro "totalmente insatisfeito”. Após a obtenção dos escores médios, a mediana dividiu a amostra nos grupos dos "mais satisfeitos" e "menos satisfeitos".

Das 667 pessoas sorteadas, foram excluídas 18 pessoas com HAS que não faziam tratamento com medicamentos ou eram gestantes com doença hipertensiva da gravidez. Houve 54 perdas: não foram localizadas 19 pessoas e 35 não consentiram na participação. Foram estudadas, então, 595 pessoas cujas características foram apresentadas em outro estudo 12 .

Os dados foram obtidos mediante entrevistas pessoais (com cerca de uma hora de duração) realizadas na residência das pessoas que consentiram em participar do estudo por entrevistadores previamente treinados, no período compreendido entre março e agosto de 2006. Todos os entrevistados assinaram Termo de Consentimento Livre e Esclarecido.

As entrevistas foram revisadas pelos pesquisadores para controle de qualidade do preenchimento. Foram consultados os prontuários dos voluntários nas unidades de saúde para esclarecer ou complementar informações. Os dados foram digitados em banco de dados eletrônico (aplicativo Epidata 3.0. Epidata Association, Odense, Dinamarca).

As variáveis de estudo foram descritas por freqüência absoluta e freqüência relativa. Para variáveis quantitativas, foram calculadas as medidas de tendência central (média e mediana) e de dispersão (desvio padrão). Foi calculada a prevalência de não-adesão com intervalo de 95\% de confiança (IC95\%) para uma distribuição binomial.

Objetivou-se testar a hipótese de que a nãoadesão (variável dependente) está associada a 
múltiplos fatores hierarquicamente ordenados (Figura 1). Considerou-se que condições sócioeconômicas (bloco 1) estão associadas à nãoadesão de maneira direta ou mediada por características dos serviços de saúde (bloco 2), ou ainda por características imediatamente atribuíveis às pessoas e ao seu tratamento medicamentoso (bloco3).

Calculou-se OR (odds ratio), com IC95\%, como medida de associação entre a variável de desfecho "não-adesão" (variável categórica definida pelo QAM-Q) e cada variável independente (análise univariada). Utilizou-se o teste de quiquadrado de Pearson para estabelecer a significância estatística. Para variáveis ordinais, a significância estatística foi estimada pelo teste de quiquadrado de tendência. No estudo de associação com variáveis quantitativas, utilizou-se do teste $\mathrm{t}$ de Student não pareado para comparação entre médias 13

$\mathrm{Na}$ análise multivariada, calcularam-se os valores de OR por regressão logística não condicional, hierarquizados por blocos de variáveis. De início, procedeu-se à modelagem do bloco 1 para ajuste das variáveis sócio-econômicas, utilizando-se das variáveis do mesmo bloco. A seguir, construiu-se outro modelo para ajustar as variáveis relativas aos serviços de saúde, utilizando as variáveis do mesmo bloco. Este modelo foi ajustado pelo conjunto de variáveis ajustadas no bloco anterior. Um terceiro modelo, do bloco das características das pessoas e dos medicamentos, foi realizado para ajustar as variáveis internamente ao bloco. Em seguida, tal modelo foi ajustado pelo conjunto de blocos antecedentes.

No processo de modelagem de cada bloco, foram incluídas todas aquelas variáveis que obtivessem um valor de $\mathrm{p}<0,10$ na análise univariada. Utilizou-se a técnica de retirada paulatina das variáveis com base nos níveis de significância (stepwise backward), permanecendo aquelas que mantiveram um valor de $\mathrm{p}<0,05$ para o estabelecimento de significância estatística dos modelos, estimado pelo Teste da Razão de Máxima Verossimilhança.

O estudo foi feito em conformidade com a Declaração de Helsinki e aprovado pela Comissão de Ética para Análise de Projetos de Pesquisa do Hospital das Clínicas, Faculdade de Medicina, Universidade de São Paulo, de acordo com o parecer constante do processo de $n^{\circ} .878 / 05$.

\section{Resultados}

Com base na medida combinada do QAM-Q, 316 pessoas foram consideradas não aderentes, re- presentando uma prevalência de não adesão de 53,1\% (IC95\%: 49,0-57,2).

A média de idade dos aderentes (62,1 anos) foi superior à média de idade das pessoas não aderentes (59,2 anos) ( $\mathrm{p}<0,001)$.

Pessoas que se declararam brancas tiveram em média mais tempo de estudo, 3,76 anos, do que não brancas com 3,08 anos ( $p<0,05)$, assim como as pessoas de classe A/B (6,0 anos) comparadas com aquelas que pertenciam às classes $\mathrm{C} / \mathrm{D} / \mathrm{E} \operatorname{com} 3,2$ anos $(\mathrm{p}<0,001)$.

A média de idade das pessoas que trabalhavam era menor do que as que não trabalhavam, respectivamente, 50,4 e 63,9 anos ( $p<$ 0,0001). Pessoas inseridas no mercado de trabalho apresentaram maior tempo desde a última consulta quando comparadas àquelas que não trabalhavam, respectivamente, 121,6 e 85,5 dias $(\mathrm{p}<0,01)$.

Encontrou-se associação estatisticamente significante entre tabagismo e consumo de álcool $(\mathrm{p}<0,01)$. O hábito de fumar e consumo de álcool se mostraram inversamente associados com idade $(\mathrm{p}<0,01)$.

As pessoas classificadas como aderentes tomavam, em média, mais medicamentos que os não aderentes, respectivamente 4,8 e 4,1 medicamentos $(\mathrm{p}<0,001)$. O tempo médio de tratamento foi menor em pessoas classificadas como não aderentes (119,4 meses), comparado com os aderentes $(137,6$ meses) $(\mathrm{p}<0,05)$.

Destaca-se a presença de transtornos mentais comuns em 265 dos entrevistados $(44,8 \%$, IC95\%: 40,5-48,6), os quais apresentavam tempo médio desde a última consulta menor que os demais (75,2 dias e 110,6 dias, respectivamente $\mathrm{p}<0,001)$.

Interrupção prévia se mostrou associada à média de idade mais baixa (56,1 x 62,3 anos, $\mathrm{p}<0,001)$ e com relato de reações adversas $(\mathrm{p}<0,01)$. As que relataram reações adversas tomavam, em média, mais medicamentos do que aqueles que não relataram (5,2 e 4,4, respectivamente $(\mathrm{p}<0,01)$, e eram mais freqüentes entre aquelas que tomavam 3 ou mais anti-hipertensivos $(\mathrm{p}<0,02)$.

Expressar maior satisfação para com os serviços se mostrou associado com ausência de transtornos mentais comuns $(\mathrm{p}<0,001)$.

Dentre as variáveis sócio-econômicas, escolaridade, renda individual e condições de moradia não apresentaram associação estatisticamente significante com não-adesão. No bloco das variáveis relativas aos serviços de saúde, comparecimento a consultas ou a grupos, e procurar hospital no último ano também não se mostraram associadas à não-adesão. Dentre as características das pessoas, sexo, estado civil, religião, 
consumo atual de tabaco e álcool não estavam associadas à não-adesão. Por fim, do bloco das variáveis relativas a doenças e medicamentos, aquelas sem associação foram: número de antihipertensivos, esquema terapêutico, necessitar de ajuda para tomar os medicamentos, uso de remédios caseiros, presença de reações adversas e outras doenças associadas.

A análise univariada das associações que se mostraram estatisticamente significantes entre variáveis de estudo e a não-adesão são apresentadas na Tabela 1 .

A Tabela 2 apresenta os modelos de regressão logística hierarquizada que ajustam os valores de OR brutos resultante da análise univariada, seguido dos valores ajustados pelas variáveis do mesmo bloco e dos blocos hierarquicamente superiores.

\section{Discussão}

O subgrupo de pessoas inseridas no mercado de trabalho, em especial aqueles trabalhadores não qualificados e com baixo poder aquisitivo (classes C, D e E) apresenta maior risco de não-adesão, como já observado em outros estudos 14,15. Ressalte-se que a inserção no mercado de trabalho está associada com idade menor, menos tempo de tratamento e maior tempo desde a última consulta, condições que parecem mediar sua associação à não-adesão 16 .

As associações verificadas entre classe econômica $\mathrm{C} / \mathrm{D} / \mathrm{E}$ e baixa renda individual, baixa escolaridade e cor não branca sugerem que essas variáveis se apresentam como indicadores representativos da condição sócio-econômica. Encontrou-se também associação entre classes econômicas e tipo de serviço utilizado (classes A/B utilizaram mais serviços particulares e convênios), o que pode explicar parte do processo de mediação desta variável com a não-adesão.

A baixa escolaridade tem se mostrado associada à não-adesão 17 e ao abandono ao tratamento 18, o que não foi observado neste estudo. Isso pode ser explicado, ao menos em parte, pela associação da escolaridade com diversas outras variáveis sócio-econômicas que podem ter tido melhor poder discriminatório.

As variáveis relativas aos serviços de saúde sugerem que pessoas que necessitam comprar seus medicamentos e que tiveram sua última consultahámais deseis meses apresentam risco maior de não-adesão, mesmo quando ajustados pelos fatores sócio-econômicos presentes no bloco 1 .

No caso da fonte de medicamentos, os valores de OR observados na análise univariada sofreram um pequeno aumento após o ajuste pelas variáveis do mesmo bloco e das variáveis sócioeconômicas. Esta variação pode representar um ajuste dos fatores sócio-econômicos. Contudo, é importante ressaltar que o número pequeno de pessoas que relataram ter de comprar seus medicamentos pode prejudicar sua participação no modelo logístico.

Alguns estudos mencionam a falta de dinheiro para comprar os remédios como fator explicativo para a não-adesão 19,20 ou, ainda, que a renda familiar elevada esteja associada à maior adesão ${ }^{14}$. Neste estudo, a não-adesão também é maior entre aqueles que precisam comprar a medicação.

$\mathrm{O}$ acesso a medicamentos tem sido uma das pedras angulares para o controle da hipertensão arterial 2. O Município de Blumenau possui na sua Relação Municipal de Medicamentos todas as classes de anti-hipertensivos (exceto bloqueadores de receptor AT1 da angiotensina II). A pequena proporção de pessoas (menos que $4 \%$ ) que relata ter de comprar seus medicamentos, comparada com o número que consulta médico particular ou de plano de saúde, parece refletir uma prática reconhecida de pessoas (mesmo de baixa renda) buscarem atendimento em consultório particular, mas obterem seus medicamentos no SUS.

O maior risco estimado de não-adesão entre os que tiveram consultas há mais de seis meses pode refletir problemas de acesso a consultas. De outro lado, pessoas mais jovens, inseridas no mercado de trabalho (sejam como empregadas ou autônomas) e que não possuem transtorno mental comum apresentaram maior tempo desde a última consulta, o que pode estar relacionado com dificuldades no reconhecimento da necessidade de assistência médica.

Há, porém, controvérsias quanto à associação entre freqüência de consultas, adesão e mesmo controle clínico, o que pode indicar que a qualidade das consultas pode ser mais relevante do que a freqüência 21,22.

Em relação às características das pessoas, mantiveram-se no modelo final as variáveis: transtornos mentais comuns, ter interrompido o tratamento anteriormente, tempo de tratamento e número de medicamentos consumidos. Estas duas últimas foram as que sofreram maior ajuste pelos blocos de variáveis sócio-econômicas e assistenciais, e a última perdeu significância quando ajustada.

Resultados de diversos estudos com pessoas hipertensas reiteram que quanto menor o tempo de doença (e de tratamento farmacológico) maior a não-adesão e o abandono ${ }^{23}$. A hipertensão arterial é uma doença crônica insidiosa, de longa duração, na maior parte do tempo assintomática, 
Tabela 1

Análise univariada da associação de não-adesão e variáveis de estudo.

\begin{tabular}{|c|c|c|c|c|c|}
\hline Variável de estudo & Aderentes & Não aderentes & OR & IC95\% & Valor de $p$ \\
\hline \multicolumn{6}{|l|}{ Bloco 1: sócio-econômicas } \\
\hline Raça/Cor & & & & & 0,01 \\
\hline Branca & 237 & 243 & 1,0 & & \\
\hline Preta ou Outras & 42 & 73 & 1,7 & $1,1-2,6$ & \\
\hline Grupo ocupacional & & & & & $<0,001$ \\
\hline Industriários & 75 & 59 & 1,0 & & \\
\hline Agricultores & 28 & 25 & 1,1 & $0,6-2,2$ & \\
\hline Chefias/Comércio/Serviços & 152 & 321 & 1,4 & $0,9-2,1$ & \\
\hline Trabalhadores braçais não qualificados & 24 & 87 & 3,3 & $1,9-6,0$ & \\
\hline Status ocupacional & & & & & 0,03 \\
\hline Sem trabalhar & 222 & 227 & 1,0 & & \\
\hline Trabalhando & 57 & 89 & 1,5 & $1,0-2,2$ & \\
\hline \multicolumn{6}{|l|}{ Classe econômica } \\
\hline $\mathrm{A} / \mathrm{B}$ & 53 & 40 & 1,0 & & 0,04 \\
\hline$C / D / E$ & 226 & 276 & 1,6 & $1,0-2,5$ & \\
\hline \multicolumn{6}{|l|}{ Bloco 2: serviços de saúde } \\
\hline Tempo da última consulta (meses) & & & & & 0,04 \\
\hline Até 6 & 238 & 245 & 1,0 & & \\
\hline Mais que 6 & 39 & 64 & 1,6 & $1,0-2,5$ & \\
\hline \multicolumn{6}{|l|}{ Tipo de serviço médico } \\
\hline Particular/Convênio & 57 & 47 & 1,0 & & \\
\hline SUS & 222 & 269 & 1,5 & $1,0-2,3$ & 0,05 \\
\hline Fonte dos medicamentos & & & & & 0,02 \\
\hline SUS & 275 & 300 & 1,0 & & \\
\hline Precisou comprar & 4 & 16 & 3,7 & $1,2-11,1$ & \\
\hline \multicolumn{6}{|l|}{ Bloco 3: Características pessoais } \\
\hline Faixa etária (anos) & & & & & 0,01 * \\
\hline 70 e mais & 83 & 69 & 1,0 & & \\
\hline $50-69$ & 153 & 178 & 1,4 & $1,0-2,1$ & \\
\hline Até 49 & 43 & 69 & 1,9 & $1,2-3,2$ & \\
\hline Interrompeu tratamento? & & & & & $<0,01$ \\
\hline Não & 216 & 205 & 1,0 & & \\
\hline $\operatorname{Sim}$ & 61 & 108 & 1,9 & $1,3-2,7$ & \\
\hline Satisfação com serviço & & & & & 0,03 \\
\hline Mais satisfeitos & 165 & 162 & 1,0 & & \\
\hline Menos satisfeitos & 102 & 145 & 1,5 & $1,0-2,0$ & \\
\hline \multicolumn{6}{|l|}{ Bloco 3: doença e medicamentos } \\
\hline Tempo de tratamento (anos) & & & & & 0,01 \\
\hline Mais de 3 & 231 & 232 & 1,0 & & \\
\hline Até 3 & 46 & 82 & 1,8 & $1,2-2,7$ & \\
\hline Número de medicamentos & & & & & $<0,01 *$ \\
\hline 5 ou mais & 136 & 124 & 1,0 & & \\
\hline $3-4$ & 92 & 101 & 1,2 & $0,8-1,7$ & \\
\hline $1-2$ & 51 & 91 & 2,0 & $1,3-3,0$ & \\
\hline Transtorno mental comum & & & & & $<0,01$ \\
\hline Ausente & 174 & 152 & 1,0 & & \\
\hline Presente & 102 & 163 & 1,8 & $1,3-2,5$ & \\
\hline
\end{tabular}

* Qui-quadrado de tendência.

IC95\%: intervalo de 95\% de confiança; OR: odds ratio. 
Associação de não-adesão e variáveis de estudo ajustadas no modelo de regressão logística não condicional por bloco de variáveis.

\begin{tabular}{|c|c|c|c|}
\hline Variável de estudo & $\begin{array}{l}\text { OR bruto } \\
\text { (IC95\%) }\end{array}$ & $\begin{array}{l}\text { OR ajustado } \\
\text { (IC95\%)* }\end{array}$ & $\begin{array}{l}\text { OR ajustado } \\
(\text { IC } 95 \%) * *\end{array}$ \\
\hline \multicolumn{4}{|l|}{ Bloco 1: sócio-econômicas } \\
\hline \multicolumn{4}{|l|}{ Grupo Ocupacional } \\
\hline Industriários & 1,0 & 1,0 & \\
\hline Agricultores & $1,1(0,6-2,2)$ & $1,2(0,6-2,3)$ & - \\
\hline Chefias/Comércio/Serviços & $1,4(0,9-2,1)$ & $1,5(1,0-2,2)$ & \\
\hline Trabalhadores braçais não qualificados & $3,3(1,9-6,0) * * *$ & $3,2(1,8-5,8) * \star \star$ & \\
\hline \multicolumn{4}{|l|}{ Status ocupacional } \\
\hline Sem trabalhar & 1,0 & 1,0 & \\
\hline Trabalhando & $1,5(1,0-2,2) * \star \star$ & 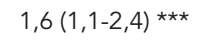 & \\
\hline \multicolumn{4}{|l|}{ Classe econômica } \\
\hline A/B & 1,0 & 1,0 & \\
\hline C/D/E & $1,6(1,0-2,5) * \star \star$ & 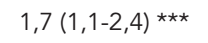 & \\
\hline \multicolumn{4}{|l|}{ Bloco 2: serviços de saúde } \\
\hline \multicolumn{4}{|l|}{ Tipo de serviço médico } \\
\hline Particular/Convênio & 1,0 & 1,0 & 1,0 \\
\hline SUS & $1,5(1,0-2,3)$ & 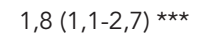 & $1,5(1,0-2,4)$ \\
\hline \multicolumn{4}{|l|}{ Fonte dos medicamentos } \\
\hline SUS & 1,0 & 1,0 & 1,0 \\
\hline Precisou comprar & $3,7(1,2-11,2)$ *** & $4,9(1,6-15,3)$ *** & $4,5(1,4-14,0)$ *** \\
\hline \multicolumn{4}{|l|}{ Tempo da última consulta (meses) } \\
\hline Até 6 & 1,0 & 1,0 & 1,0 \\
\hline 6 e mais & $1,6(1,0-2,5) * \star \star$ & $1,7(1,1-2,6) * \star \star$ & 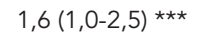 \\
\hline \multicolumn{4}{|l|}{ Bloco 3: doença e medicamentos } \\
\hline \multicolumn{4}{|l|}{ Interrompeu o tratamento } \\
\hline Não & 1,0 & 1,0 & 1,0 \\
\hline $\operatorname{Sim}$ & $1,9(1,3-2,7) * \star \star$ & 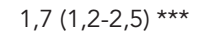 & $1,8(1,2-2,7) * \star \star$ \\
\hline \multicolumn{4}{|l|}{ Número de medicamentos } \\
\hline 5 ou mais & 1,0 & 1,0 & 1,0 \\
\hline $3-4$ & $1,2(0,8-1,7)$ & $1,2(0,8-1,7)$ & $1,2(0,8-1,8)$ \\
\hline $1-2$ & $2,0(1,3-3,0) * * *$ & 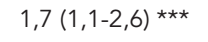 & $1,5(0,9-2,4)$ \\
\hline \multicolumn{4}{|l|}{ Tempo de tratamento (anos) } \\
\hline Mais de 3 & 1,0 & 1,0 & 1,0 \\
\hline Até 3 & 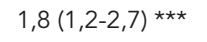 & $1,6(1,0-2,4) * \star \star$ & 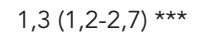 \\
\hline \multicolumn{4}{|l|}{ Transtorno mental comum } \\
\hline Ausente & 1,0 & 1,0 & 1,0 \\
\hline Presente & $1,8(1,3-2,5) * \star \star$ & $1,9(1,3-2,6) * \star \star$ & $2,1(1,4-2,9) * \star \star$ \\
\hline
\end{tabular}

IC95\%: intervalo de 95\% de confiança; OR: odds ratio.

* OR ajustado pelas variáveis do mesmo bloco;

** OR ajustado pelas variáveis do mesmo bloco e blocos anteriores;

*** $p<0,05$.

fato que induz muitas pessoas a não se reconhecerem como doentes. Além disso, dependendo da situação clínica, existe a não-indicação de terapêutica medicamentosa e, quando há, muitos a recusam ou a abandonam rapidamente. A percepção de melhora com o tratamento também pode levar à interrupção. Assim, neste estudo, embora a variável "interromper previamente o tratamento" possa refletir dificuldades de acesso, o pequeno ajuste observado sugere mais fortemente problemas de não-reconhecimento da doença. 
A presença de transtornos mentais se manteve fortemente associada à não-adesão, após ajuste pelas variáveis do mesmo bloco e pelos blocos anteriores. Alguns autores afirmam que a presença de sintomas depressivos, bem como a perda de função cognitiva se mostram associadas à não-adesão após ajuste para variáveis sócio-demográficas 24,25 .

Menor número de medicamentos consumidos associou-se com não-adesão, tendo sofrido ajuste pelas variáveis do mesmo bloco (pessoas com menos idade e menos tempo de tratamento tomavam menos medicamentos) e perdido significância no modelo ajustado pelas variáveis de blocos anteriores. É possível que expresse o fato de pessoas com mais idade, com mais tempo de tratamento e melhores condições sócioeconômicas e assistenciais, além de consumirem mais medicamentos, tratam de consumi-los com maior adesão, inclusive os anti-hipertensivos.

Este estudo apresenta três possíveis limitações que merecem destaque. Primeira, o processo amostral pode ter sofrido viés de seleção, pois as pessoas com HAS foram identificadas com base no cadastro elaborado pelos agentes comunitários de saúde das unidades amostradas. Algumas pessoas com hipertensão podem não ter sido cadastradas (em especial, quadros limítrofes ou os pacientes que não se reconhecem como hipertensos, bem como pessoas ativas no mercado de trabalho). Todavia, é licito supor que, caso tenha ocorrido de fato este viés, o mesmo foi no sentido de diminuir a associação encontrada porque os resultados obtidos na análise univariada sugeriram associação entre pessoas que trabalham e não-adesão. Os resultados sugerem também que pessoas somente com HAS tinham maior associação com não-adesão, se comparados com pessoas com HAS e doenças associadas (inclusive diabetes mellitus). Assim, o possível excesso na inclusão de pessoas nessas categorias (aposentados ou com diabetes) poderia levar a uma diminuição das medidas de efeito obtidas.

Segunda, considera-se classicamente que o uso de questionários subestima a não-adesão. Uma alternativa é aumentar o ponto de corte de não-adesão como forma de melhorar a sensibilidade. O uso da medida combinada do QAM-Q aumenta a sensibilidade por considerar aderente apenas quem tomou seus medicamentos na quantidade correta, de modo correto e que relata efeito favorável.

Terceira, os estudos revisados utilizam métodos diversos para medir a adesão, estimando dimensões distintas do fenômeno. Assim, as associações observadas em diversos fatores de risco podem manifestar-se em algumas situações, mas não em outras. Ainda que se tenha tomado o cuidado de levar em conta tal diversidade, isso pode ter limitado a comparabilidade dos resultados obtidos.

O modelo obtido neste estudo pode subsidiar o estabelecimento de estratégias para diminuir barreiras sócio-econômicas (trabalho, qualificação profissional e poder aquisitivo), melhorar o acesso a consultas e medicamentos bem como identificar grupos de pessoas com maior risco de não-adesão.

Aos profissionais e gestores da atenção primária, em particular da Estratégia Saúde da Família, fica o desafio de propor e executar abordagens coletivas (grupos, campanhas etc.) e individuais (nas consultas médicas, na enfermagem e nas visitas dos agentes de saúde) para melhorar a adesão. Isso pode materializar-se na busca ativa de unidades por pessoas com baixa freqüência às unidades, bem como a ampliação de fornecimento de medicamentos (entrega domiciliar, por exemplo) para quem tem limitação de locomoção ou trabalha durante todo o dia. A implantação de equipes multiprofissionais de apoio (Núcleos de Apoio à Saúde da Família - NASF), especialmente com profissional farmacêutico, também pode contribuir para melhorar a adesão.

Melhorar a adesão não é tarefa fácil. Revisão sistemática recente concluiu que intervenções para melhorar a adesão (educativas, comportamentais ou baseadas em recursos tecnológicos) apresentam resultados limitados e precisam ser desenvolvidas e adaptadas às características das pessoas e contextos dos serviços 26 . O desenvolvimento de uma intervenção para melhorar a adesão - intervenção que esteja baseada na reflexão e respeito à autonomia e individualidade do paciente - e sua avaliação em estudos adequados (do tipo ensaio clínico) devem estar presentes na agenda dos pesquisadores. 


\section{Resumo}

Para estimar a prevalência e analisar fatores associados à não-adesão ao tratamento de pessoas com hipertensão arterial sistêmica, atendidas em unidades de saúde da família, procedeu-se a estudo transversal com 595 pacientes. A variável dependente não-adesão foi medida com questionário (Questionário de Adesão a Medicamentos - QAM-Q). Foram coletadas variáveis sócio-econômicas, assistenciais, pessoais e do tratamento, analisadas por modelo de regressão logística hierarquizado. A prevalência de não-adesão foi de 53\%. As variáveis associadas à não-adesão foram: (1) sócio-econômicas - pertencer às classes econômicas C/D/E, estar inserido no mercado de trabalho, em ocupações não qualificadas; (2) assistenciais - precisar comprar os medicamentos e mais que 6 meses desde a última consulta, e; (3) características das pessoas e do tratamento - interromper previamente o tratamento, estar em tratamento há menos de 3 anos e presença de transtorno mental comum. O estudo dos determinantes da não-adesão articulados em um modelo hierarquizado sugere que as desigualdades sociais se mostram diretamente associadas à não-adesão, ou mediadas por fatores dos serviços e das pessoas.

Hipertensão; Adesão à Medicação; Atenção Primária à Saúde; Estudos Epidemiológicos

\section{Colaboradores}

E. T. Santa-Helena e M. I. B. Nemes contribuíram na concepção do projeto, análise e interpretação dos dados, redação do artigo e revisão crítica relevante do conteúdo intelectual e aprovação final da versão a ser publicada. J. Eluf Neto colaborou na análise e interpretação dos dados, revisão crítica relevante do conteúdo intelectual e aprovação final da versão a ser publicada.

\section{Agradecimentos}

À equipe Qualiaids (Departamento de Medicina Preventiva, Faculdade de Medicina, Universidade de São Paulo) pelos debates, sugestões e críticas.

\section{Referências}

1. Pereira M, Lunet N, Azevedo A, Barros H. Differences in prevalence, awareness, treatment and control of hypertension between developing and developed countries. J Hypertens 2009; 27:963-75.

2. Passos VMA, Assis TD, Barreto SM. Hipertensão arterial no Brasil: estimativa de prevalência a partir de estudos de base populacional. Epidemiol Serv Saúde 2006; 15:35-45.

3. Ministério da Saúde. Hipertensão arterial sistêmica para o Sistema Único de Saúde. Brasília: Ministério da Saúde; 2006. (Cadernos de Atenção Básica, 16).

4. World Health Organization. Adherence to longterm therapies: evidence for action. Geneva: World Health Organization; 2003
5. Krousel-Wood MA, Muntner P, Islam T, Morisky DE, Webber LS. Barriers to and determinants of medication adherence in hypertension management: perspective of the Cohort Study of Medication Adehrence Among Older Adults. Med Clin N Am 2009; 93:753-69.

6. Prado JC, Kupek E, Mion Jr. D. Validity of four indirect methods to measure adherence in primary care hypertensives. J Hum Hypert 2007; 21:579-84.

7. Ungari AQ. Adesão ao tratamento farmacológico de pacientes hipertensos seguidos nos Núcleos de Saúde da Família do município de Ribeirão Preto, SP [Dissertação de Mestrado]. Ribeirão Preto: Universidade de São Paulo; 2007. 
8. Victora CG, Huttly SR, Fuchs SC, Olinto MTA. The role of conceptual frameworks in epidemiological analysis: a hierarchical approach. Int J Epidemiol 1997; 26:224-7.

9. Santa-Helena ET, Nemes MIB, Eluf Neto J. Desenvolvimento e validação de questionário para medir não-adesão ao tratamento com medicamentos. Rev Saúde Pública 2008; 42:764-7.

10. Mari JJ, Williams P. A validity study of a Psychiatric Screening Questionnaire (SRQ-20) in primary care in the city of São Paulo. Br J Psychiatry 1986;148:23-6.

11. Daneluz M, Costa LH, Gewehr C, Santa-Helena ET, Nemes MIB. Desenvolvimento e validação de questionário de satisfação dos usuários hipertensos do Programa de Saúde da Família, Blumenau, SC. In: Anais do VIII Congresso Brasileiro de Saúde Coletiva. Rio de Janeiro: ABRASCO; 2006.

12. Santa-Helena ET, Nemes MIB, Eluf Neto J. Avaliação da assistência a pessoas com hipertensão arterial atendidas em unidades de saúde da família. Saúde Soc 2010; no prelo.

13. Altman R. Practical statistics for medical research. London: Chapman \& Hall; 1991.

14. DiMatteo MR. Variations in patients' adherence to medical recommendations: a quantitative review of 50 years of research. Med Care 2004; 42:200-9.

15. Teixeira ACA. Adesão ao tratamento farmacológico da hipertensão arterial e seus determinantes em pacientes de ambulatório [Dissertação de Mestrado]. Fortaleza: Universidade Federal do Ceará; 1998.

16. Wroth TH, Pathman DE. Primary medication adherence in a rural population: the role of the patient-physician relationship and satisfaction with care. J Am Board Fam Med 2006; 19: 478-86.

17. Yiannakopoulou EC, Papadopulos JS, Cokkinos DV, Mountokalakis TD. Adherence to antihypertensive treatment: a critical factor for blood pressure control. Eur J Cardiovasc Prev Rehabil 2005; 12:243-9.

18. Busnello RG, Melchior R, Faccin C, Vettori D, Petter J, Moreira LB, et al. Characteristics associated with the dropout of hypertensive patients followed up in an outpatient referral clinic. Arq Bras Cardiol 2001; 76:352-4.
19. Soumerai SB, Pierre-Jacques M, Zhang F, Ross-Degnan D, Adams AS, Gurwitz J, et al. Cost-related medication nonadherence among elderly and disabled medicare beneficiaries. Arch Intern Med 2006; 166:1829-35.

20. Wilson J, Axelsen K, Tang S. Medicaid prescription drug access restrictions: exploring the effect on patient persistence with hypertension medications. Am J Manag Care 2005; 11 Espec No.:SP27-34.

21. Strelec MAAM, Pierin AMG, Mion Jr, D. The influence of patient's consciousness regarding high blood pressure and patient's attitude in face of disease controlling medicine intake. Arq Bras Cardiol 2003; 81:349-54

22. Coelho EB, Moyses Neto M, Palhares R, Cardoso MCM, Geleilete TJM, Nobre F. Relação entre a assiduidade às consultas ambulatoriais e o controle da pressão arterial em pacientes hipertensos. Arq Bras Cardiol 2005; 85:157-61.

23. Jokisalo E, Kumpusalo E, Enlund H, Halonen P, Takala J. Factors related to non-compliance with anti-hypertensive drug therapy. J Hum Hypert 2002; 16:577-83.

24. DiMatteo MR, Giordani PJ, Lepper HS, Croghan TW. Patient adherence and medical treatment outcomes: a meta-analysis. Med Care 2002; 40:794811.

25. Wang PS, Bohn RL, Knight E, Glynn RJ, Mogun H, Avorn J. Noncompliance with antihypertensive medications: the impact of depressive symptoms and psychosocial factors. J Gen Intern Med 2002; 17:504-11.

26. Haynes RB, Ackloo E, Sahota N, McDonald HP, Yao X. Interventions for enhancing medication adherence. Cochrane Database Syst Rev 2008; (2):CD000011.

Recebido em 08/Fev/2010

Versão final reapresentado em 21/Jun/2010

Aprovado em 11/Ago/2010 